\title{
Erratum to: A dynamic VM consolidation technique for QoS and energy consumption in cloud environment
}

\author{
Seyed Yahya Zahedi Fard ${ }^{1}$ (D) Mohamad Reza Ahmadi ${ }^{2} \cdot$ \\ Sahar Adabi ${ }^{3}$
}

Published online: 1 June 2017

(C) Springer Science+Business Media New York 2017

\section{Erratum to: J Supercomput DOI 10.1007/s11227-017-2016-8}

The authors very much regret that errors have slipped into their contribution.

The corrections are listed below.

Sahar Adabi's affiliation was incorrect. The correct affiliation is given here.

\subsection{Virtual machine selection}

The subsection should read:

There are more than one virtual machines run on a server; thus, it needs to consider how to choose a migratable virtual machine when a server is overloaded. Different parameters involve to select the virtual machine, and at the moment, there are four types of virtual machine selection policies: maximum correlation (MC), minimum

The online version of the original article can be found under doi:10.1007/s11227-017-2016-8.

Seyed Yahya Zahedi Fard

Yahya.zahedi@gmail.com

Mohamad Reza Ahmadi

M.ahmadi@itrc.ac.ir

Sahar Adabi

Sahar_adabi@iau-tnb.ac.ir

1 Department of Computer Engineering, Islamic Azad University Science and Research Branch, Tehran, Iran

2 Department of Information Technology, Iran Telecommunication Research Center, Tehran, Iran

3 Department of Computer Engineering, Islamic Azad University North Tehran Branch, Tehran, Iran 
migration time (MMT), minimum utilization (MU), and random selection (RS) [19]. MC migrates a virtual machine that has the maximum correlation coefficient compared to the other virtual machines on the same server. The MMT migrates those virtual machines that will take the least time to move. MU selects a virtual machine with the lowest utilization. And, RS migrates virtual machines randomly without any rules. We propose a novel virtual machine selection algorithm which calculates the deviation between utilization of overloaded server and its threshold and find the virtual machine in which virtual machine utilization is close to the deviation. We named it maximum fit (MF) because we wanted to find maximum fit virtual machines for migration; in other words, we want to get a server back to the normal state with the minimum number of live migration. We find the virtual machine by binary search in all the virtual machines on overload server to decrease the number of migrations to prevent performance degradation due to live migration. The general algorithm of virtual machine selection is shown in Algorithm 3. The complexity of algorithm is log $n$, where $\mathrm{n}$ is the number of virtual machine in overloaded server.

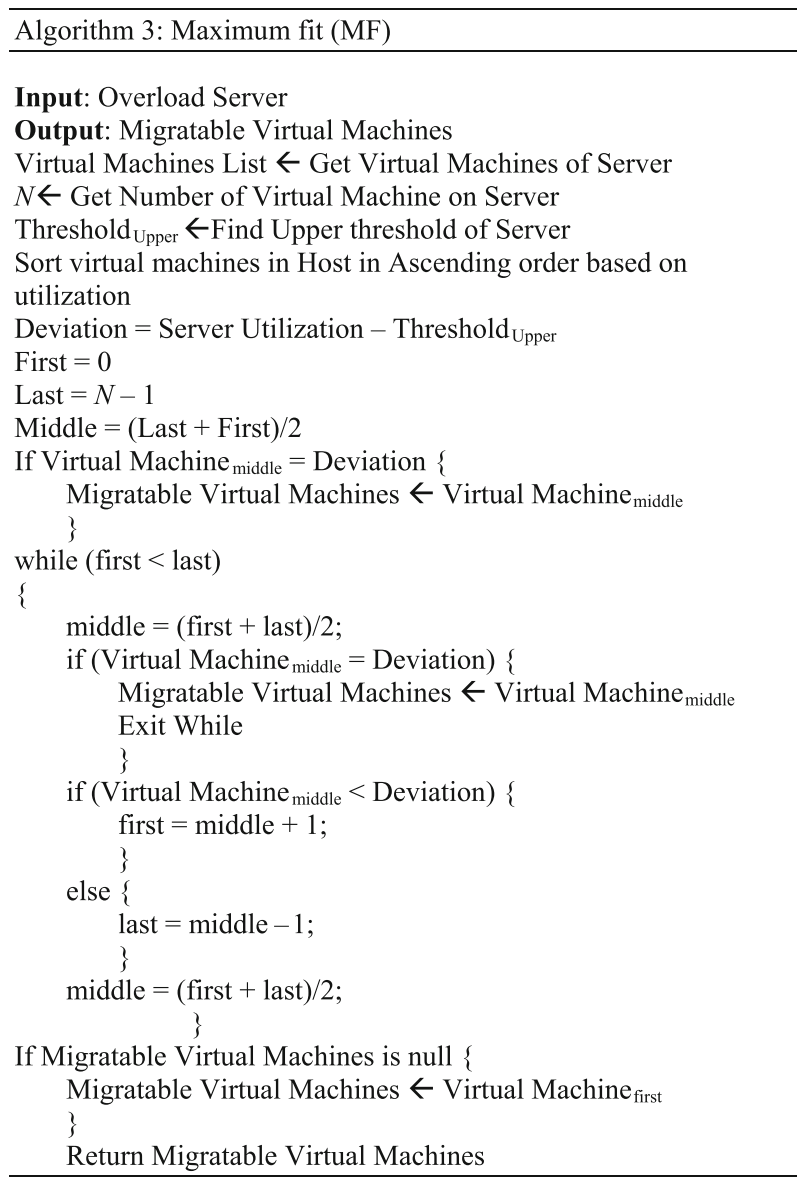




\subsection{Virtual machine placement}

The subsection should read:

After selecting virtual machines that need to be migrated, determining servers that host those virtual machines is the last steps of virtual machine consolidation. One method used in [8] is the Power-Aware Best Fit Decreasing (PABFD); it allocates each virtual machine to a server that provides the least increase in power consumption due to this allocation. We mentioned in Sect 4.2. A number of server type consumes more energy consumption against the other while doing less operation per second, and we attempt to select such servers to improve energy consumption of servers, decrease the number of active servers, and aim to decrease the number of low-category servers, but here is vice versa. We propose a new algorithm to allocate virtual machines to the server that select the server within high-category servers to preserve highcategory server active and low-category servers inactive. The core idea behind this is the low-category servers consume more energy consumption per operation against the other. Thus, if we placed virtual machine to this server, it improves the energy consumption of server and aims to select a high-category server in advance. The goal of this algorithm is to allocate virtual machines to the server that provides the least increase and has more resource capacity for virtual machine placement in future. The core idea behind this is the more resource capacity, the less probability of overload occurrence which can lead to fewer resource shortages and consequent decrease in migrations.

We have done our test on different servers with different characteristics to examine our idea, and the result shows that if we select a high-category server that provides the least increase in power consumption due to this allocation, it decreases live migration and accordingly performance degradation due to live migration and SLA violation in comparison with selecting low-category server. The general algorithm of the server under load detection is shown in Algorithm 4. $N$ is the number of server category, and greater $N$ means the high-performance server with less energy consumption. The complexity of algorithm is $n m+n=O(n m)$, where $m$ is the number of servers in specific type and $n$ is a constant that indicates the number of server type; in this paper, we selected $n$ as 3 (low, medium, and high) values, so the complexity equal to $3 m+3=O(m)$. 


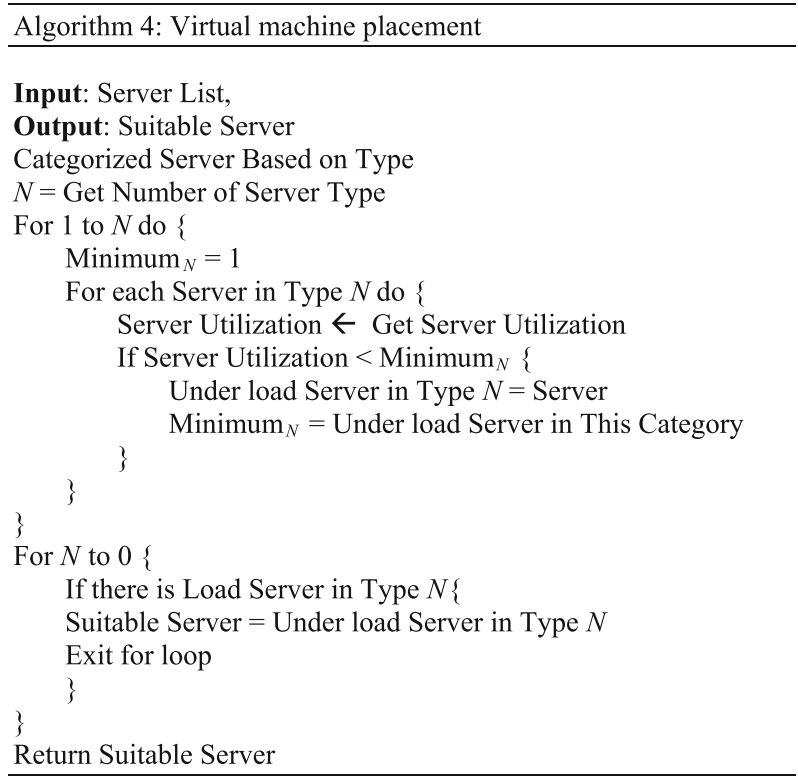

